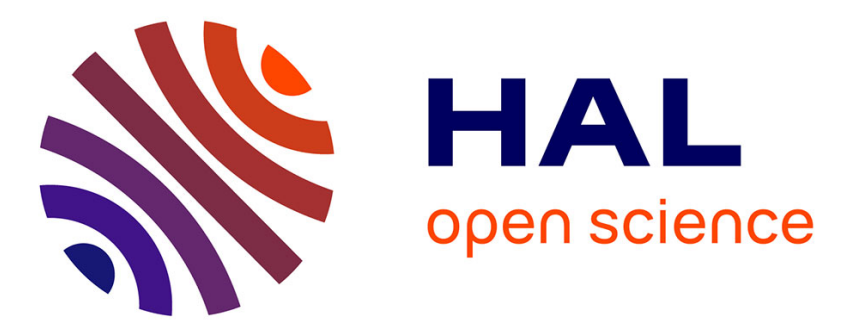

\title{
A Synthetic-Vision Based Steering Approach for Crowd Simulation
}

\author{
Jan Ondrej, Julien Pettré, Anne-Hélène Olivier, Stéphane Donikian
}

\section{To cite this version:}

Jan Ondrej, Julien Pettré, Anne-Hélène Olivier, Stéphane Donikian. A Synthetic-Vision Based Steering Approach for Crowd Simulation. ACM Transactions on Graphics, 2010, SIGGRAPH 2010 Papers, 4 (123), 10.1145/1778765.1778860 . inria-00539572

\section{HAL Id: inria-00539572 \\ https://hal.inria.fr/inria-00539572}

Submitted on 24 Nov 2010

HAL is a multi-disciplinary open access archive for the deposit and dissemination of scientific research documents, whether they are published or not. The documents may come from teaching and research institutions in France or abroad, or from public or private research centers.
L'archive ouverte pluridisciplinaire HAL, est destinée au dépôt et à la diffusion de documents scientifiques de niveau recherche, publiés ou non, émanant des établissements d'enseignement et de recherche français ou étrangers, des laboratoires publics ou privés. 


\section{A Synthetic-Vision Based Steering Approach for Crowd Simulation}

\author{
Jan Ondřej* \\ INRIA
}

\author{
Julien Pettré* \\ INRIA
}

Anne-Hélène Olivier*
INRIA

\author{
Stéphane Donikian* \\ INRIA
}

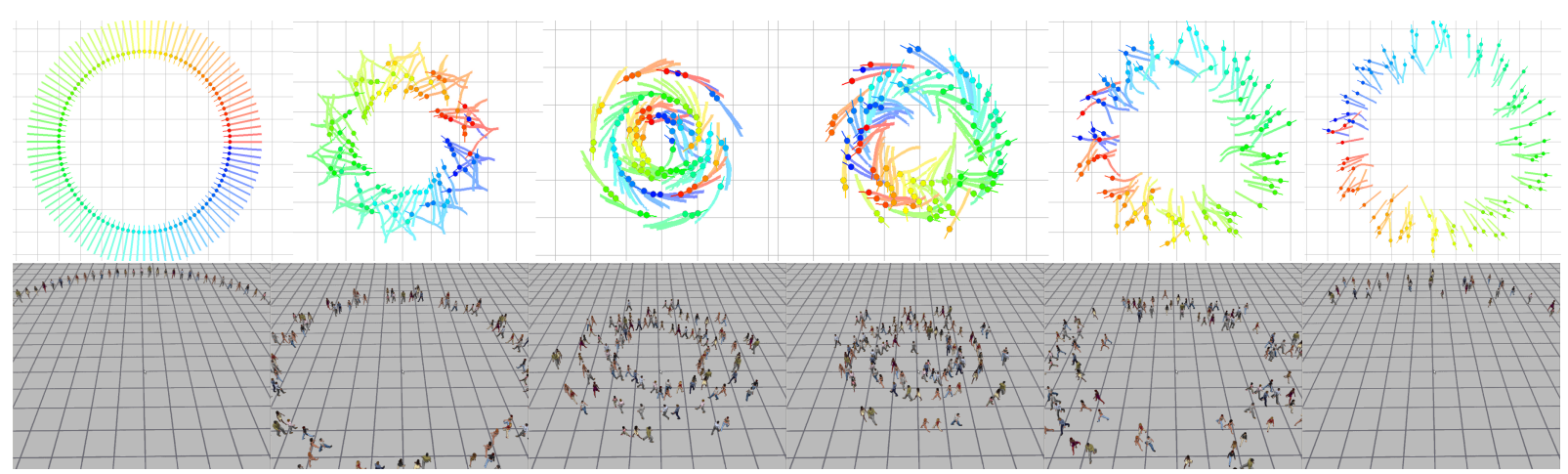

Figure 1: Animations resulting from our simulations. Emergent self-organized patterns appear in real crowds of walkers. Our simulations display similar effects by proposing an optic flow-based approach for steering walkers inspired by cognitive science works on the human locomotion. Compared to previous approaches, our model improves such an emergence as well as the global efficiency of walkers traffic. We thus enhance the overall believability of animations by avoiding improbable locking situations.

\section{Abstract}

In the everyday exercise of controlling their locomotion, humans rely on their optic flow of the perceived environment to achieve collision-free navigation. In crowds, in spite of the complexity of the environment made of numerous obstacles, humans demonstrate remarkable capacities in avoiding collisions. Cognitive science work on the human locomotion stated that a relatively succinct information is extracted from the optic flow to achieve a safe locomotion. In this paper, we explore a novel vision-based approach of collision avoidance between walkers that fit the requirements of interactive crowd simulation. In imitation of humans and based on cognitive science results, we detect future collisions as well as their dangerousness from visual-stimuli. The motor-response is twofold: reorientation strategy is set to avoid future collision, whereas a deceleration strategy is used to avoid imminent collisions. Several examples of our simulation results show that the emergence of self-organized patterns of walkers is reinforced using our approach. Emergent phenomena are visually appealing. More importantly, they improve the overall efficiency of the walkers traffic and allow avoiding improbable locking situations.

CR Categories: I.3.7 [Computer Graphics]: Three-Dimensional Graphics and Realism-Animation I.6.5 [Simulation and Modeling]: Types of Simulation-Animation

Keywords: crowd simulation, steering method, collision avoidance, synthetic vision

\section{Introduction}

Crowd simulation has significantly grown in importance these two past decades. Their field of application is wide and ranges from the domains of security and architecture to the one of movie industry and interactive entertainment. The visually impressive selforganized patterns that emerge at a large-scale from the combination of all the local actions and interactions in crowds is probably a major reason of the attention paid by Computer Animation on this topic. Reynolds' seminal work on flocks of boids showed that fascinating global motions can be obtained from simple local interactions rules [Reynolds 1987]; however, the proposed rules explicitly stick boids together to obtain emerging flocks. Moreover, boids motion rules are not directly transposable to human walkers.

Human crowds are the place of numerous and various interactions. In this paper, we focus on crowds of individually walking humans where interactions are limited to collision avoidance. Our motivation is to design a local collision avoidance method that remains as close as possible to the real human behavior while displaying emerging self-organized patterns as witnessed in real crowds. This objective is representative of our bottom-up approach: specific large-scale formations are expected from realistic local interactions between walkers. Simulating emerging formations is crucial in order to obtain believable crowd animations. Obtaining them from individually steered walkers avoiding each-other, and thus, simulating self-organization, is particularly challenging.

\footnotetext{
*e-mail:\{jan.ondrej,julien.pettre,anne-helene.olivier,donikian\}@irisa.fr
} 
Collision avoidance has recently received much attention. Several types of approach were proposed (cf. Section 2 for an overview). Most of recent agent-based techniques are based on geometrical models. Their common point is to explicitly compute admissible velocities that allow avoiding future collisions: efforts are focused on reaching highest performance in order to handle large crowds. Then, challenge is to steer walkers with believable trajectories while remaining in the admissible velocity domain. However, geometrical models are also disconnected from reality since humans unconsciously react to perceived obstacles to avoid collisions. This raises fundamental question, that is can simpler perception/action control loops - probably closer to reality - steer virtual walkers and allow them avoiding collisions even in complex situations? Rule-based techniques explored such a question; however, artifacts occur in most complex situations because of the difficulty in combining rules. Particle-systems and continuum-based methods ease the combination of interactions and are able to handle even larger crowds. They however have drawbacks as well. The former sometimes fail to simulate emerging patterns of walkers while the latter may lead to unrealistic local motions as for example unfeasible accelerations or velocities.

In contrast with previous approaches, we steer walkers according to the visual perception they have of their environment. We thus formulate our collision avoidance solution as a visual-stimuli/motorresponse control law. Our model is inspired by the work of Cutting and colleagues [1995] on the human locomotion in the field of cognitive science. They stated that humans extract two major elements from their optic flow to achieve collision-free navigation. First is the derivative of the bearing-angle under which obstacles are perceived. Second is the time-to-collision which is deduced from the rate of growth of obstacles in successively perceived images. Inspired by these observations, our model's inputs, i.e., the visual-stimuli, are the egocentrically perceived obstacles transformed into images of time-derivatives of bearing-angles and of times-to-collision. These images are directly computed from the geometries and states of both the static and moving obstacles of the scene. Walkers have simple reactions to these stimuli: they turn to avoid future collisions and decelerate in the case of imminent collisions.

Our contributions are thus the following. We propose a visionbased collision avoidance model for interactive simulation of crowds of individual humans. We base our approach on cognitive science work on the human locomotion, which inspired us novel local visual-stimuli/motor-response laws. We apply our method to complex situations of interaction: resulting simulations display the emergence of interesting self-organized patterns of walkers at a global-scale. We demonstrate our improvements in comparison to previous approaches, with enhanced emergence of patterns of walkers, improved global efficiency of the walkers traffic, and smoother animations.

The remainder of the paper is organized as follows. Section 2 first provides an overview of crowd simulation techniques with particular focus on collision avoidance methods. Following, we present the guiding principles of our approach before describing the proposed model in details in Section 3. We provide details about its implementation in Section 4. Finally, we illustrate simulation results from several examples and give comparison with previous techniques in Section 5. Limitations of our approach and future work are discussed in Section 6, before concluding.

\section{Related Work}

Virtual Crowd is a wide topic that raises numerous problems including population design, control, simulation or rendering and was surveyed in recent books [Thalmann and Raupp Musse 2007; Pelechano et al. 2008] and tutorials [Thalmann et al. 2005; Halperin et al. 2009]. This overview focuses on crowd simulation, the objective of which can be restrictively defined as computing global locomotion trajectories to achieve goal-driven collision-free navigation for crowds of walkers.

Several classes of solutions were proposed in the literature. Cellular-automaton approaches [Schadschneider 2001] are used to simulate evacuation scenarios for large crowds: the discrete aspect of resulting trajectories prevent their use for Computer Animation applications. However, grid-based solutions were adapted to meet such requirements [Loscos et al. 2003], and for example Shao and Terzopoulos [2005] proposed the use of multi-resolution grids to handle large environments. Other techniques consider velocity-fields to guide crowds [Chenney 2004]. This analogy with Physics gave rise to particle-systems approaches. Helbing [1995] proposed the social-forces model where walkers repulse each-other while they are attracted by their goal. The social forces model was later revisited in [Pelechano et al. 2007; Gayle et al. 2009]. Evolved models proposed using mass-damp-spring systems to compute similar repulsing forces between walkers [Heigeas et al. 2003]. Crowd simulation was also studied as a flowing continuum [Hughes 2003; Treuille et al. 2006] that allows simulating numerous walkers in real-time. Even larger crowds were handled using hybrid continuum-based approach [Narain et al. 2009]. From a general point-of-view, high computation performance is a common point between all of these approaches. Such performance allows simulating large crowds in equally large environments in real-time, which is a crucial need of many interactive applications. Performance is however obtained at the cost of some limitations, such as restricting the total number of goals walkers can have, or using of simplistic interaction models that may lower the realism of results. Compared to this former set of approaches, our first objective is not to reach a high-performance solution but to simulate local interactions in a realistic manner. By realism, we here mean that we reproduce the human vision-based locomotion control in order to steer walkers in crowds. Synthetic vision raises numerous computations by nature.

Our method can be closely related to rule-based approaches [Reynolds 1999] as well as to geometrically-based local avoidance models approaches [Paris et al. 2007; van den Berg et al. 2008; Kapadia et al. 2009; Pettré et al. 2009; Karamouzas et al. 2009; Guy et al. 2009]. It is generally required to combine local approaches with dedicated techniques in order to enable the reaching of high-level goals in complex environments [Lamarche and Donikian 2004; Paris et al. 2006; Pettré et al. 2006; Sud et al. 2007]. Nevertheless, geometrically-based avoidance models carefully check the absence of future collisions locally, given the simulation state. This goal is generally achieved by decomposing the reachable velocity-space of each walker into two components: the inadmissible velocity domain and the admissible velocity domain. These domains respectively correspond to velocities leading to collisions and those allowing avoidance. At the opposite, our method make walkers react to some situations without explicitly computing the admissibility of their motion adaptations. This raises a fundamental question: can explicit collision checks guarantee the absence of residual collisions? We argue the answer is negative. The reason is twofold. First, the admissible velocity domain is computed assuming that the velocity of moving obstacles remains constant. Second, the admissible velocity domain is often made of several independent components, especially in the case of complex interactions - i.e., during simultaneous interactions with several obstacles. Some of these components degenerate in time because moving obstacles may also adapt their own motion. If the current velocity of a given walker belong such a degenerative component, switching to another component is required. As a 
result, traversing the inadmissible velocity domain is required when acceleration is bounded, whereas unbounded accelerations result into unrealistic motions. Our method do not explicitly check collisions and is not exempt from failure. We however believe the proposed visual-stimuli/motor-response laws better imitate the most basic level of real human locomotion control.

We previously addressed the question of realism of simulated locomotion trajectories during collision avoidance in [Pettré et al. 2009]. We provide a qualitative description of such trajectories: we experimentally show that real humans anticipate avoidance as no more adaptation is required some seconds before walkers pass at close distance. We also show that avoidance is a role-dependent behavior as the walker passing first makes noticeably less adaptations than the one giving way. We discuss the visual information humans may exploit to be able to achieve avoidance in such a manner. However, we proposed a geometrical model to reproduce such trajectories that is calibrated from our experimental dataset. Compared to this work, we here address two new problems. First, we address the question of combining interactions. We explore synthetic vision as a solution to implicitly combine them, for example: they are integrated by projection to the perception image, they are filtered when obstacles are invisible, they are weighted by the importance obstacles have in the image. Second, we directly base our motion control laws on the visual information believed to be exploited by real humans.

Vision-based methods were never used to tackle the crowd simulation problem to the best of our knowledge, with the exception of Massive software agents [Massive ] which are provided with synthetic vision; however, controlling walkers from such an input is left at the charge of users. Nevertheless, synthetic vision was used to steer a single or few virtual humans [Noser et al. 1995; Kuffner and Latombe 1999; Peters and O'Sullivan 2003] or artificial creatures [Tu and Terzopoulos 1994]. Reynolds' boids were also recently provided with visual perception abilities [Silva et al. 2009]. Our approach explores a new type of visual-stimuli to control locomotion, based on statements from cognitive science. We also improve performance to fit the requirements of interactive crowd simulation. Finally, visual-servoing is an active topic in the field of Robotics [Chaumette and Hutchinson 2006]. Major challenges are processing optic flows acquired with physical systems and extracting the relevant information that allow steering robots. In contrast to this field, we do not process digitally computed images but directly compute the required visual-inputs of our model.

\section{Vision-based collision avoidance}

\subsection{Model overview}

Humans control their locomotion from their vision [Warren and Fajen 2004]. According to Cutting and colleagues [Cutting et al. 1995] humans successively answer two questions during interactions with static and moving obstacles: will a collision occur? When will collision occur? Cutting experimentally observed that these two questions are answered by extracting two indicators from the perceived optic flow:

1. Will a collision occur? Humans visually perceive obstacles under a given angle referred to as the bearing-angle (noted $\alpha$ ). A collision is predicted when the time derivative of the bearing angle, $\dot{\alpha}$, is zero (or close to zero because of the body envelopes). This observation is illustrated in Figure 2 from the 3 examples of two walkers displaying converging trajectories.

2. When will collision occur? Humans visually perceive obstacles with given sizes. The rate-of-growth of obstacles in time

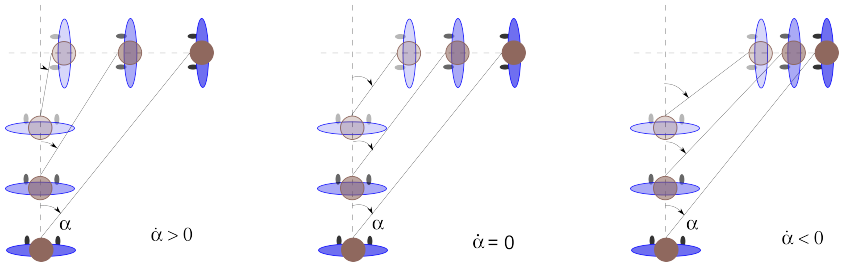

Figure 2: The bearing-angle and its time-derivative, respectively $\alpha$ and $\dot{\alpha}$, allow detecting future collisions. From the perspective of an observer (the walker at the bottom), a collision is predicted when $\alpha$ remains constant in time. (left) $\alpha<0$ and $\dot{\alpha}>0$ : the two walkers will not collide and observer will give way. (center) the bearingangle is constant $(\dot{\alpha}=0)$. The two walkers will collide. (right) $\alpha<0$ and $\dot{\alpha}<0$ : the two walkers will not collide and observer will pass first.

allow humans to detect obstacles coming toward them when positive. Moreover, the higher the rate the more imminent the collision. As a result, humans are able to evaluate the time-tocollision $(t t c)$.

Therefore, the relevant information necessary to achieve collisionfree locomotion according to Cutting is entirely described by the pair $(\dot{\alpha}, t t c)$. It is to notice that humans use similar information to intercept mobile targets as described by Tresilian in [Tresilian 1994].
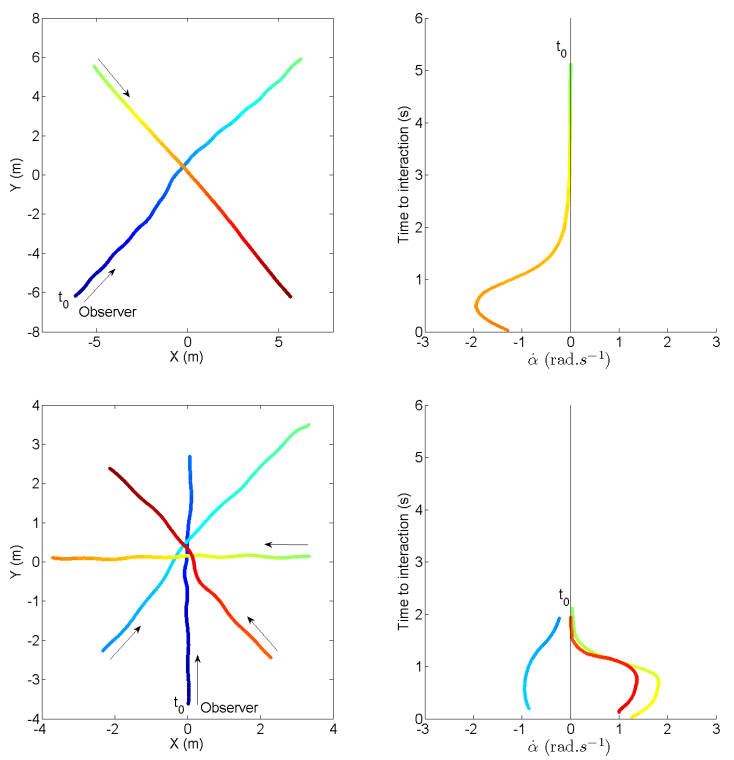

Figure 3: Two examples of real interactions between (top) two walkers and (bottom) four walkers. Motion captured trajectories projected on the ground are shown (plots on the left), as well as in the $(\dot{\alpha}, t t i)$-space (plots on the right), as perceived by one of the participant called 'observer'. Trajectories are colored in order to enable matching between the two representations.

Figure 3 illustrates Cutting's theory from 2 examples of real interactions: trajectories are displayed in the horizontal plane as well as in the $(\dot{\alpha}, t t i)$-space, where $t t i$ is the time-to-interaction. Time-tointeraction is the time remaining before minimum distance between participants is reached, according to current positions and velocities. The notion of time-to-collision $t t c$ is generally used in the literature in place of our time-to-interaction $t t i$; these two notions are 
close. By definition ttc exists if and only if a risk of future collision is also existing. At the opposite, $t t i$ exists whatever the relative positions and velocities of the considered moving objects. Also note that $t t i$ can reach negative values when the considered objects display diverging motions. In the first example, we observe that $\dot{\alpha}$ is initially close to zero whilst $t t i$ decreases: collision is predicted. By turning to the left, the observer solves the interaction: $\dot{\alpha}$ decreases. On the second example, future collision with the observer is predicted for two walkers among the three perceived ones. By turning and decelerating, $\dot{\alpha}$ values are corrected. The impact of motion adaptations on the variations of $(\dot{\alpha}, t t i)$ is not intuitive. However, as a first approximation, turns mainly plays on the $\dot{\alpha}$ value, whereas a deceleration mainly changes $t t i$.

The guiding principles of the proposed model - based on Cutting's results - are thus the following. A walker perceives the static and moving obstacles of his environment as a set of points $P=\left\{p_{i}\right\}$ resulting from his synthetic vision. For each perceived point $p_{i}$, we compute the bearing angle $\alpha_{i}$, its time-derivative $\dot{\alpha}_{i}$, and the remaining time-to-interaction relatively to the walker $t t i_{i}$. We deduce the risk of a future collision from $\dot{\alpha}_{i}$. We also deduce the dangerousness of the situation from $t t i_{i}$. A walker reacts when needed according to two strategies. First, he avoids future collision by adapting his orientation with anticipation. Second, in the case of an imminent collision, he decelerates until he gets stopped or the interaction is solved. The following sections detail how we put these principles into practice.

\subsection{Model inputs}

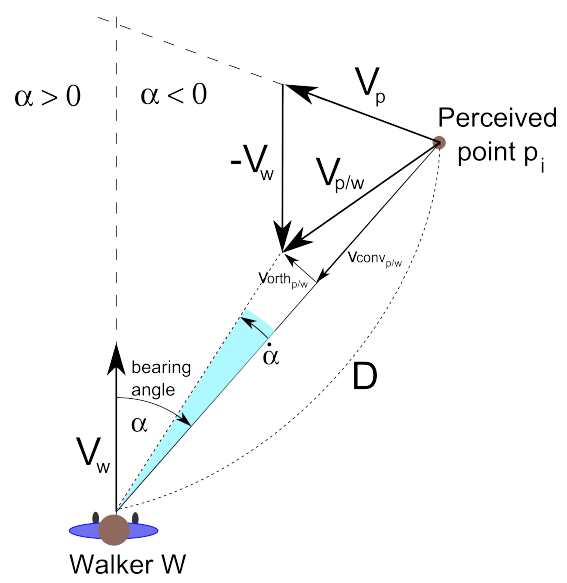

Figure 4: Model's inputs. Any point is perceived under given bearing-angle. The triad $\left(\alpha_{i}, \dot{\alpha}_{i}, t t i_{i}\right)$ is deduced from the relative point position and velocity with respect to the walker.

A walker configuration is defined by its position and orientation $\theta$. He is velocity-controlled by his angular velocity $\dot{\theta}$ and his tangential velocity $v$. Perceived points $p_{i} \in P$ may indiscriminately belong to static obstacles - such as walls - or moving ones - such as other walkers. Also note that a single obstacle result in several points with respect to its shape: Figure 6 illustrates how a walker perceives his environment. The variables associated to each $p_{i} \rightarrow\left(\alpha_{i}, \dot{\alpha}_{i}, t t i_{i}\right)$ are deduced from the relative position and velocity of $p_{i}$ to the walker; we however detail their computation in Figure 4 as well as in the following Implementation Section 4.

\subsection{Angular velocity control}

As explained in the previous section, a walker detects a risk of future collision when $\dot{\alpha}$ is low and $t t i_{i}>0$. We define the $\dot{\alpha}_{i}$ thresh- old $\tau_{1}$ under which a walker reacts as a function of the perceived $t t i_{i}$ as follows:

$$
\tau_{1}(t t i)= \begin{cases}\tau_{1-}(t t i)=a-b . t t i^{-c} & \text { if } \dot{\alpha}_{i}<0, \\ \tau_{1+}(t t i)=a+b . t t i^{-c} & \text { otherwise. }\end{cases}
$$

where $a, b$ and $c$ are some parameters of the model. These three parameters change a walker avoidance behavior by adapting his anticipation time as well as the security distance he maintains with obstacles. We detail the role of these parameters in the Discussion Section 6. Figure 5 plots the function $\tau_{1}$ for $a=0, b=0.6$ and $c=1.5$. These values were used in the examples shown in Section 5 , and were determined by manually fitting $\tau_{1}$ on numerous experimental data capturing avoidance between real walkers similar to those shown in Figure 3 . Then, the set $P_{c o l}$ of points $p_{i}\left(\dot{\alpha}_{i}, t t i_{i}\right)$ a walker has to react to is defined as follows:

$$
p_{i} \in P_{c o l} \text { if } t t i_{i}>0 \text { and } \alpha_{i}<\tau_{1}\left(t t i_{i}\right)
$$

We now combine the influence of the set of points belonging to $P_{c o l}$. For this purpose, we decompose $P_{c o l}$ into $P_{+}$and $P_{-}$, which respectively correspond to points with positive and negative $\dot{\alpha}_{i}$ values. We then define $\phi_{+}$and $\phi_{-}$as follows:

$$
\begin{array}{cc}
\phi_{+}=\min \left(\dot{\alpha}_{i}-\tau_{1+}\left(t t i_{i}\right)\right), & \text { for all } p_{i} \in P_{+} \\
\phi_{-}=\max \left(\dot{\alpha}_{j}-\tau_{1-}\left(t t i_{j}\right)\right), & \text { for all } p_{j} \in P_{-}
\end{array}
$$

At this point, we have identified all interactions requiring walkers to turn to the right to avoid future collision into $P_{+}$, and those asking to turn left into $P_{-}$. The required amplitude of a right turn allowing to avoid at once all the interactions provoked by the $P_{+}$set of points directly depends on the amplitude of $\phi_{+}$(the same for a left turn, $P_{-}$and $\phi_{-}$respectively). However, we must ensure walkers do not highly deviate from their goal. For this reason, we now consider the bearing-angle corresponding to the goal $\alpha_{g}$, as well as its timederivative $\dot{\alpha}_{g}$. Contrarily to obstacles, walkers attempt to intercept their goal, which means that $\dot{\alpha}_{g}=0$ is desired. Three cases are then successively considered. Firstly, when $\dot{\alpha}_{g}$ is small (we arbitrarily choose $\left|\dot{\alpha}_{g}\right|<0.1 \mathrm{rad} . \mathrm{s}^{-1}$ ), walkers are currently heading to their goal, the influence of which is neglected. In this case, we simply choose the change of direction which asks the minimum deviation. $\dot{\theta}$ is controlled as follows:

$$
\dot{\theta}= \begin{cases}\phi_{+} & \text {if }\left|\phi_{+}\right|<\left|\phi_{-}\right|, \\ \phi_{-} & \text {otherwise. }\end{cases}
$$

Secondly, when $\phi_{-}<\dot{\alpha}_{g}<\phi_{+}$, but cannot be neglected, we choose the change of direction that leads to the smallest deviation from the goal. Then,

$$
\dot{\theta}= \begin{cases}\phi_{+} & \text {if }\left|\phi_{+}-\dot{\alpha}_{g}\right|<\left|\phi_{-}-\dot{\alpha}_{g}\right| \\ \phi_{-} & \text {otherwise. }\end{cases}
$$

Thirdly, when $\dot{\alpha}_{g}<\phi_{-}$or $\dot{\alpha}_{g}>\phi_{+}$we choose:

$$
\dot{\theta}=\dot{\alpha}_{g}
$$

To avoid unrealistic angular velocities, $\dot{\theta}$ and $\ddot{\theta}$ are finally bounded so that $|\dot{\theta}|<\pi / 2\left(\right.$ rad.s $\left.^{-1}\right)$ and $|\ddot{\theta}|<\pi / 2\left(\right.$ rad.s $\left.^{-2}\right)$.

\subsection{Tangential velocity control}

Tangential velocity $v$ is set to comfort velocity $v_{\text {comf }}$ by default. It is only adapted in the case of a risk of imminent collision. The imminence of a collision is detected when $t t i_{i}$ is positive but lower 


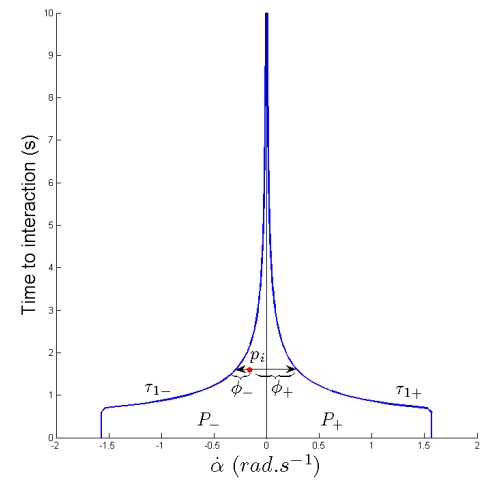

Figure 5: $\tau_{1}$ plot using the following parameter set: $a=0, b=$ 0.6 and $c=1.5$ ( $c f$. Equation (1)). Future collision is detected when $p_{i}\left(\dot{\alpha}_{i}, t t i_{i}\right)$ is below $\tau_{1}$ and $t i_{i}>0$. The plot also illustrates that the lower the tti $i_{i}$ value, the higher the walker's reaction.

than a threshold $\tau_{2}$ (we arbitrarily choose $\tau_{2}=3 \mathrm{~s}$.). Tangential velocity is controlled from the minimum positive $t t i_{m p}$ value perceived by the walker. We define $P_{\text {pos }}$ the set of points $p_{i} \in P_{c o l}$ for which $t t i_{i}<\tau_{2}$ and compute $t t i_{m p}$ as follows:

$$
t t i_{m p}=\min \left(t t i_{i}\right) \text { for all } p_{i} \in P_{p o s}
$$

Finally, the walker's tangential velocity is controlled as follows:

$$
v= \begin{cases}v_{\text {comf }} & \text { if } P_{\text {pos }}=\emptyset, \\ v_{\text {comf }} \cdot\left(1-e^{-0.5 t t i_{m p}^{2}}\right) & \text { otherwise. }\end{cases}
$$

Position and orientation of the walker are finally updated according to the computed $v$ and $\dot{\theta}$ values, with bounded $\dot{v}\left(|\dot{v}|<1 \mathrm{~m} . \mathrm{s}^{-2}\right)$.

\section{Implementation}

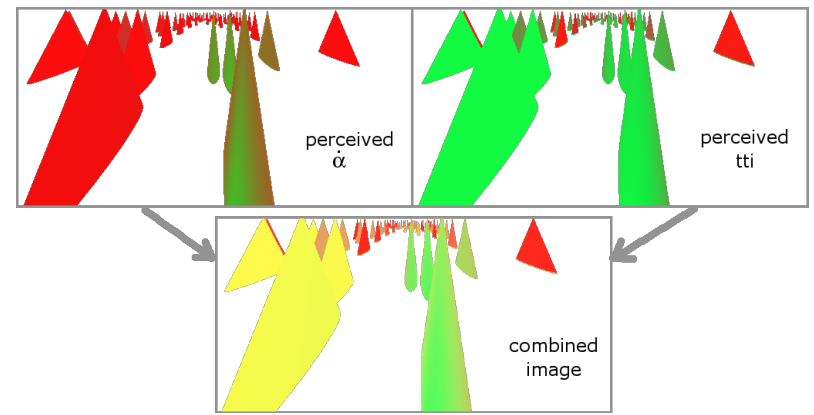

Figure 6: Walkers perceive the environment obstacles as a set of points $p_{i}\left(\dot{\alpha}_{i}, t t i_{i}\right)$. The image corresponding to all the perceived $\dot{\alpha}_{i}$ values of $p_{i}$ is shown top-left (red are for lowest values). The image corresponding to all the perceived tti $i_{i}$ values of $p_{i}$ is shown top-right (red are for lowest values). Perception is combined (bottom image) to compute walker reaction. In this example - which corresponds to the circle example, cf. Section 5 - the walker will react to the most red points of the combined perception. In this particular situation, he is likely to follow the walker in front of him on his right.

We implemented our model using OpenGL, shader programming language and CUDA. The algorithm is decomposed into two major stages. First, for each virtual walker:
Step 1 Set camera position and orientation at the one of the considered walker (see details below).

Step 2 Render to texture environment obstacles using simplified geometries. Compute values $\alpha_{i}, \dot{\alpha}_{i}$ and distance to obstacle $d$ per vertex (Figures 4 and 6)

Step 3 Then, using a fragment shader, compute per pixel $t t i_{i}$, build $P_{+}$and $P_{-}$from $\tau_{1+}$ and $\tau_{1-}$.

Step 4 Copy the resulting texture to the CUDA space and make a parallel reduction to compute $\phi_{+}, \phi_{-}$. Result is stored to an array on the GPU.

At the end of this first loop, the resulting array is downloaded once to the CPU. Then, for each walker again:

Step 5 Compute $\dot{\alpha}_{g}$ and deduce $\dot{\theta}$ and $v$.

Step 6 Update walker's position accordingly.

Camera Setup Walkers visually perceive their environment through the OpenGL camera set at the first step of our algorithm. The camera field-of-view is $150^{\circ}$ of width and $80^{\circ}$ of height. The camera position is set at the one of the considered walker at his eye level, and panning angle is aligned on the walker's motion direction. Tilting angle is set so that the upper clipping plane is horizontal (i.e., camera is oriented toward the ground with a $-40^{\circ}$ angle). Resolution is $256 \times 48$ pixels.

Simplified Geometries The complexity of the proposed algorithm is dependent on the one of the environment (Step 2). Walker do not need to react to subtle geometrical details of the scene. Simplified bounding geometries can be used for obstacles. In particular, perceived walkers are geometrically simplified as cones of $1.8 \mathrm{~m}$ of height and $0.5 \mathrm{~m}$ of base radius. Cones, similarly to walking humans, are wider at their base than at their top. Real humans can see above others' shoulders: cones better reflect this ability than cylinders, for instance.

Computation of Inputs Model's inputs are computed as illustrated in Figure 4. In the figure, $p_{i}$ is one of the perceived points that belongs to a given obstacle $o$. The relative velocity $\vec{V}_{p_{i} / w}$ of a perceived point with respect to the considered walker are first deduced:

$$
\vec{V}_{p_{i} / w}=\vec{V}_{o}-\vec{V}_{w}
$$

where $\vec{V}_{w}$ the walker's velocity vector and $\vec{V}_{o}$ the obstacle's velocity vector the perceived point belongs to. Finally, $\vec{V}_{p_{i} / w}$ is decomposed into $\vec{V}_{c o n v_{p_{i} / w}}$ and $\vec{V}_{o r t h_{p_{i} / w}}$ to deduce $t t i_{i}$ and $\dot{\alpha}_{i}\left(\vec{V}_{c o n v}\right.$ is for the component of the relative velocity converging to the considered walker, and $\vec{V}_{\text {orth }}$ the orthogonal one):

$$
\begin{array}{r}
\vec{V}_{c o n v_{p_{i} / w}}=\left(\vec{V}_{p_{i} / w} \cdot \vec{k}\right) \cdot \vec{k} \\
\vec{V}_{\text {orth }_{p_{i} / w}}=\vec{V}_{p_{i} / w}-\vec{V}_{\text {conv }_{p_{i} / w}} \\
t t i_{i}=D \cdot\left\|\vec{V}_{c o n v_{p_{i} / w}}\right\|^{-1} \\
\dot{\alpha}_{i}=\arctan \left(\frac{\left\|\vec{V}_{\text {orth }_{p_{i} / w}}\right\|}{D-\left\|\vec{V}_{\text {conv }_{p_{i} / w}}\right\|}\right) \cdot u^{-1}
\end{array}
$$

where $D$ is the $p_{i}$-walker distance, $\vec{k}$ is the unitary $p_{i}$-walker vector, and $u$ the unit of time. 


\section{Results}

\subsection{Examples}

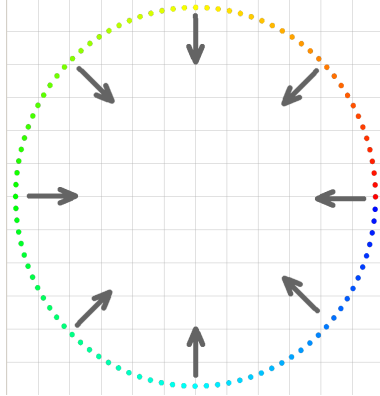

(a) Initial walkers' configuration

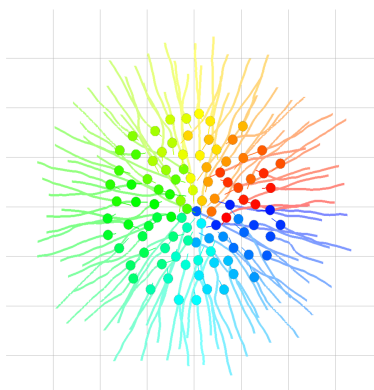

(c) RVO-Library

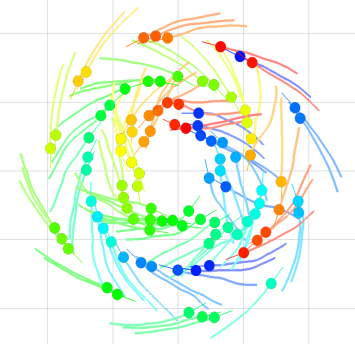

(b) Our model

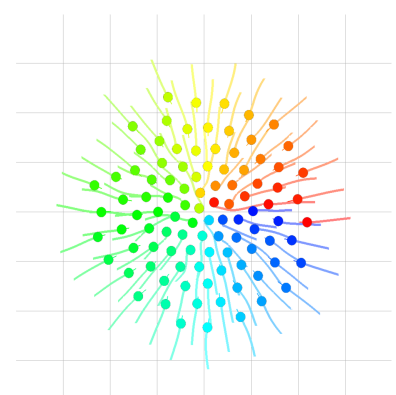

(d) Helbing's model

Figure 7: Circle (a) A scene of 100 walkers are initially deployed uniformly along a circle. Walkers goal is to reach the diametrically opposed position. Solution is shown for 3 models $(b),(c),(d)$. Our model $(b)$ is the only one able to provoke the emergence of patterns.

We illustrate our simulation results according to four examples. Comparison with two previously existing techniques is provided for the two first examples in order to illustrate the achieved improvements. We chose:

RVO which is representative of geometrical avoidance models.

Helbing's model which is representative of particle-based approaches. Contrarily to RVO, such models do not take into account anticipation and interactions are formulated as function of distance to obstacles.

The available examples are:

Circle: In this example, walkers are initially located along a circle and each one's goal is to reach the diametrically opposed position. In absence of others, each walker would go through the circle passing by its center. The number of interactions occurring in such an example is thus maximized: actually, each walker interacts with all the other ones. The main difficulty raised by this example is avoiding that walkers immediately converge to the center of the circle and get stuck there. Such situation can be efficiently avoided when 'traffic circles' emerge, whilst the center is almost left empty of anyone. Results are shown in Figure 1 and 7.

Group-swap: In this example, walkers are initially separated in two groups. The goal is to swap group positions. Motion is not constrained by static obstacles. A main difficulty raised by this example is to achieve collision avoidance whilst walkers do not excessively deviate from the shortest route in spite of

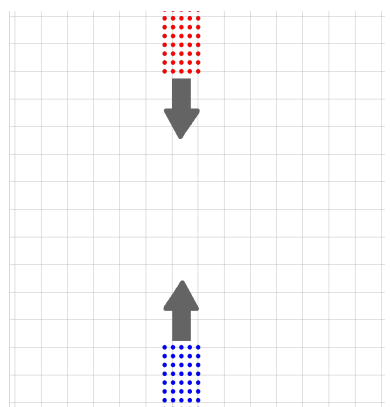

(a) Initial walkers' configuration

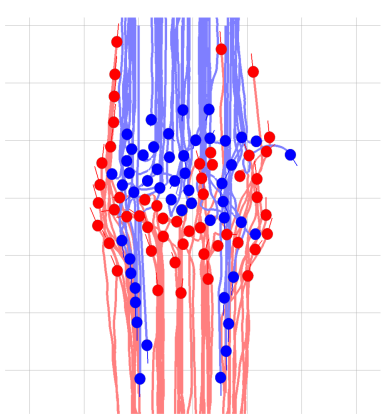

(c) RVO-Library

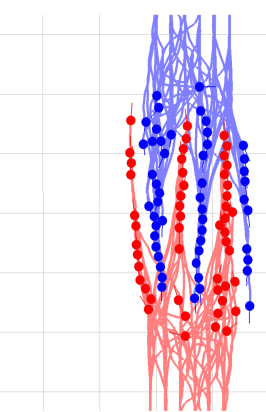

(b) Our mode

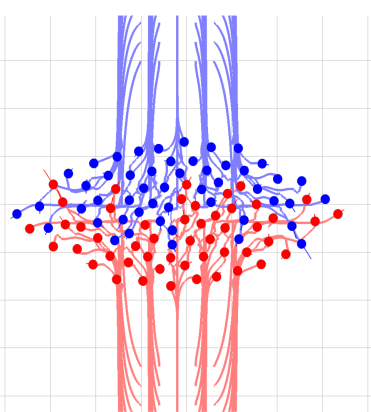

(d) Helbing's model
Figure 8: Group-swap A scene with two groups of walkers heading toward each other solved by three different models. In our model (b) distinct lane formations emerge with anticipation. The lane formations in RVO-Library (c) start emerging lately and lead to a congestion. Helbing's model (d) no such formation emerge.

the absence of constraints (e.g., corridor walls). Such a result can be reached only if lane formations emerge. Results are shown in Figure 8.

Pillars: In this example, we increase the difficulty of the groupswap example by adding two rows of pillars in the middle of the scene. We also demonstrate the ability of our model to take in account static obstacles. Results are shown in Figure 9.

Crossing: In this example, two groups of people meet at the intersection of two orthogonal corridors: static obstacles both constraints the motion and prevent walkers to early perceive the ones from the other group. Main difficulties of this example are: first, avoid that one of the two groups get stuck and second, avoid walkers to be excessively deviated along corridors walls. Results are shown in Figure 10.

All of the displayed examples demonstrate our model ability to let

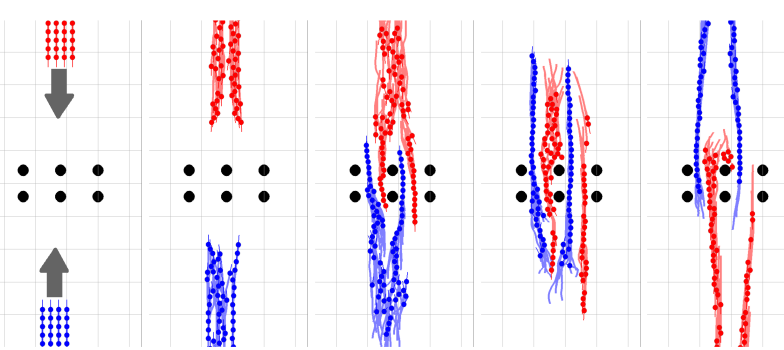

Figure 9: Pillars This example is identical to the group-swap one, two rows of pillars make the scene more complex. The images show the evolution in time of the simulation starting from the left. 


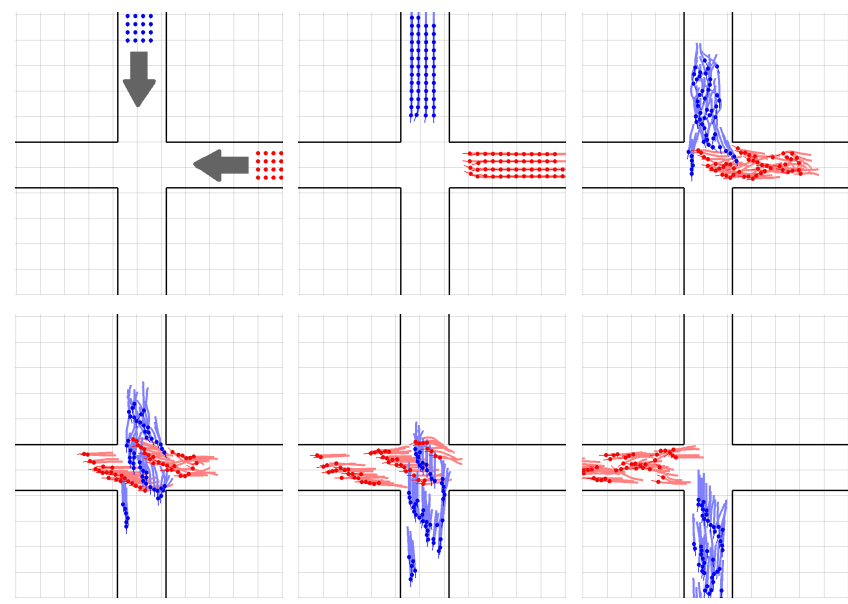

Figure 10: Crossing This example shows two groups meeting at the intersection of orthogonal corridors. The emerging line patterns the direction of which is approximately $45^{\circ}$ allows efficient global motion (evolution in time is shown from top-left to bottom-right).

self-organized patterns of walkers emerge from the motion. Emergent patterns allow to efficiently solve the sum of interactions between walkers (cf. Table 1). Improvements compared to previous approaches are perceptible: in identical situations, the walkers travel-time is lowered using our approach, and the presence of slow walkers - with $v<0.5 m . s^{-1}$ and which may affect the overall believability of results - is decreased. In the example of the circle (Figure 7), other techniques concentrate walkers in the center of the scene which lower the efficiency of the circulation. In the case of the group-swap example, the Helbing's model fail to find an acceptable solution: groups are widely spread because particles simply repulse each-other.

\begin{tabular}{|l|c|c|c|c|}
\hline \multirow{2}{*}{} & \multicolumn{2}{|c|}{ circle } & \multicolumn{2}{c|}{ group-swap } \\
\cline { 2 - 5 } & $\begin{array}{c}\text { max. } \\
\text { travel } \\
\text { time }\end{array}$ & $\begin{array}{c}\text { prop. of } \\
\text { slow } \\
\text { walkers }\end{array}$ & $\begin{array}{c}\text { max. } \\
\text { travel } \\
\text { time }\end{array}$ & $\begin{array}{c}\text { prop. of } \\
\text { slow } \\
\text { walkers }\end{array}$ \\
\hline Our model & $53 \mathrm{~s}$. & $0.97 \%$ & $55 \mathrm{~s}$. & $0.74 \%$ \\
Helbing's & $90 \mathrm{~s}$. & $30.4 \%$ & $71 \mathrm{~s}$. & $11.0 \%$ \\
RVO & $63 \mathrm{~s}$. & $13.0 \%$ & $59 \mathrm{~s}$. & $4.7 \%$ \\
\hline
\end{tabular}

Table 1: The maximum walkers travel-time and the proportion of slow walkers are provided for the circle and the group-swap examples, using three different models. The proportion of slow walkers is the mean proportion of time walkers are going below $0.5 \mathrm{~m} . \mathrm{s}^{-1}$.

Furthermore, a specificity of our model is to independently control angular and tangential velocity. Decelerations occur only in case of an imminent collision. The absence of deceleration during anticipated reaction results in smoother trajectories. We believe the overall aspect of our results is improved compared to previous approaches, especially when virtual humans are animated to follow the generated trajectories. The companion video illustrates the quality of synthetic trajectories, emergent self-organized patterns of walkers, as well as final animations.

\subsection{Performances}

Obtaining reasonable performance is probably the major technical challenge of the approach we propose due to the synthetic vision technique. We are still able to reach fair results by partly executing our algorithm steps on a GPU. Real-time performance ( 25 f.p.s.) is

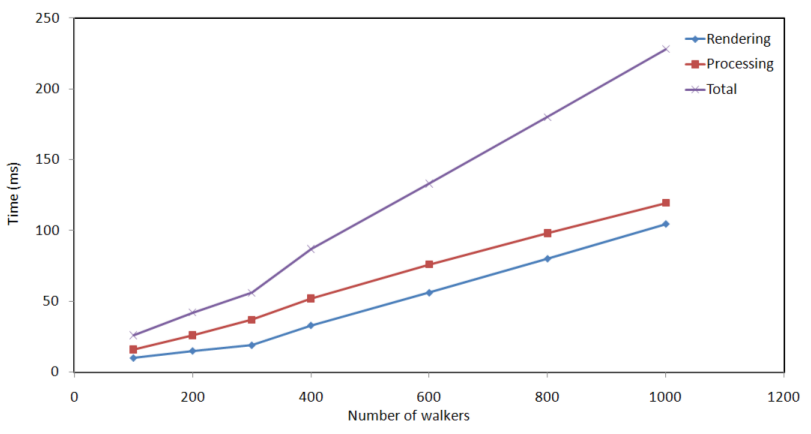

Figure 11: Performance plot: computation time for one simulation loop with respect to the number of walkers is measured. Simulation ran on a laptop with Intel T7800@2.6GHz CPU and Quadro FX $3600 M$ graphics card. The circle-example situation was used. We detail the total simulation loop time into the rendering and processing plots, which respectively correspond to the time spent during steps 1-3, and 4-6 (the latter includes the GPU-CPU data transfer).

maintained up to 200 walkers (cf. Figure 11, computed on a laptop with Intel T7800@2.6GHz CPU and Quadro FX 3600M graphics card). The major bottleneck of our method is the data transfer from the GPU to the CPU (between steps 4 and 5).

Performance can be improved in several ways. Firstly, the camera resolution at step 1 can be lowered: on one hand, the number of perceived points is decreased accordingly and performance is improved; on the other hand, perception accuracy is decreased and may prevent walkers to react with anticipation to partly occluded obstacles. The companion video illustrates the impact of lowering the camera resolution. Secondly, we believe that the complete simulation loop can be executed on the GPU (recent approaches demonstrated feasibility [Silva et al. 2009]): on one hand, data-transfer between GPU and CPU is avoided (which represent approximately $30 \%$ of the complete simulation loop time); on the other hand, further tasks could be made impossible (e.g., animating virtual walkers). Finally, assuming that each obstacle is represented by a single static or moving points (which is, for instance, an acceptable assumption for a scene made of walkers only), the model applies without need to rely on synthetic vision. On one hand, interactions are directly considered between pairs of moving points, in place of each walker and a set of perceived points. The number of processed interactions is drastically lowered. But on the other hand, synthetic vision has many advantages: the visibility of obstacles walkers react to is implicitly checked, obstacles can have any 3D shape, walkers height - which may limit their perception - is taken into account, etc.

\section{Discussion}

Realism Our results demonstrate the ability of our approach to improve the emergence of self-organized patterns of walkers on several examples. From the standpoint of Computer Animation, our method provides visually appealing results. Interactions are solved more efficiently at the global-scale: compared to other approaches, the time required for walker to reach their goal is noticeably lower using our model (cf. Table 1 and companion video for comparisons). We believe the reached efficiency benefits to the resulting believability of animations, especially, some locking situations are avoided. It is however still required to quantitatively evaluate the realism of results. Studies based on spectators feedback or, better, 
confrontation with real observations are possible directions for such an evaluation.

High-level behaviors and control Interactions between walkers are today limited to collision avoidance. Locomotion is controlled at the most basic level by visual-stimuli/motor-response laws. A near-future objective is to obtain a higher-level of control and to extend simulation abilities. Our first goal is to integrate some new types of interactions, such as following someone, reaching a mobile target, etc. Such interactions can easily be expressed in the $(\dot{\alpha}, t t i)$-space. For instance, following $p_{i}$ is controlling velocity so that $\left(\dot{\alpha}_{i} \rightarrow 0, t t i_{i} \rightarrow c s t\right)$ where $c s t$ is a positive constant. Then, our second goal is to combine different types of interactions to further improve the global efficiency of navigation by setting mid-term strategies (for instance, temporarily following someone is an efficient strategy to avoid further avoidance interactions) or to make possible the simulation of groups inside crowds (e.g., families). We assumed that goals were visible in our examples: a preliminary path planning stage would first be required to achieve navigation in complex environments. Path planners can decompose high-level goals into intermediary way-points that could be successively used as short-term goals in our model. A reactive change of short-term goals according to some external factors (e.g., local population densities) could be of interest: the evaluation future traffic conditions as well as the route selection process should be deduced from the visually perceived information in order to match our approach philosophy.

Model parameters Model's parameters $(a, b, c)$ (cf. Equation (1)) can be adapted for each walker to individualize avoidance behavior with negligible computational overhead. The impact of parameters change on simulations is illustrated in the companion video. An intuitive link exists between avoidance behavior and the shape of $\tau_{1}$ which is completely controlled by $(a, b, c)$. The higher the peak of $\tau_{1}$, the earlier the anticipation. The wider the peak, the stronger the adaptation. Finally, the curvature of $\tau_{1}$ controls a trade-off between anticipation time and reaction strength: when the maximum curvature is higher, early anticipated reactions remain low whilst they get stronger when $t t i$ decreases. The automatic adaptation of parameters with respect to external factors, such as local density of population, may open interesting perspectives.

\section{Conclusion}

We presented a novel approach of crowd simulation made of individual walkers avoiding each other. Our main contribution is to steer walkers according to the visual perception they have of their environment. We formulate their collision avoidance behavior as visual-stimuli/motor-response control laws. Compared to previous vision-based approaches, we rely on statements from cognitive science that identified the visual-stimuli humans extract from their optic flow to control their locomotion and avoid obstacles. Compared to previous avoidance models, we demonstrate our approach improves the emergence of self-organized patterns of walkers in crowd simulations. In spite of the computational complexity raised by the synthetic vision technique, we demonstrate the ability of our approach to address complex interaction situations between numerous walkers. Our results are promising and open several future work directions. First is to automatically adapt the model parameters with respect to some external factors. A second direction is to extend our model to new types of interactions. Then, our objective is to add higher level of control in order to combine several types of interactions and to enable mid-term and long-term navigation strategies. Today, the proposed approach still results into visually interesting motions that can benefit to many Computer Animation applications.
Evaluation of results by comparing them to real observations and data is now required. Nevertheless, our model is founded on cognitive science work on human locomotion which can open interesting perspectives for realistic simulation purposes.

\section{References}

Chaumette, F., And Hutchinson, S. 2006. Visual servo control, part i: Basic approaches and part ii: Advanced approaches. IEEE Robotics and Automation Magazine 13(4), 82-90.

Chenney, S. 2004. Flow tiles. In Proc. 2004 ACM SIGGRAPH/Eurographics Symposium on Computer Animation (SCA '04), Eurographics Association, Aire-la-Ville, Switzerland, Switzerland, 233-242.

Cutting, J. E., Vishton, P. M., And Braren, P. A. 1995. How we avoid collisions with stationary and moving objects. Psychological Review 102(4) (October), 627-651.

Gayle, R., Moss, W., Lin, M. C., and Manocha, D. 2009. Multi-robot coordination using generalized social potential fields. In Proc. IEEE International Conference on Robotics and Automation (ICRA '09), 106-113.

Guy, S. J., Chhugani, J., Kim, C., Satish, N., Lin, M., MANOCHA, D., AND DUBEY, P. 2009. Clearpath: highly parallel collision avoidance for multi-agent simulation. In Proc. 2009 ACM SIGGRAPH/Eurographics Symposium on Computer Animation (SCA '09), ACM, New York, NY, USA, 177-187.

Halperin, C., Anjyo, K., Cioroba, M., Kanyuk, P., Regelous, S., Yoshida, T., And Salvati, M. 2009. Crowd animation: Tools, techniques, and production examples. In $S I G$ GRAPH Asia '09: ACM SIGGRAPH Asia 2009 Courses, ACM, New York, NY, USA, 1

Heigeas, L., Luciani, A., Thollot, J., and Castagné, N. 2003. A physically-based particle model of emergent crowd behaviors. In Graphicon 2003.

Helbing, D., And Molnar, P. 1995. Social force model for pedestrian dynamics. Physical Review E 51, 4282.

Hughes, R. L. 2003. The flow of human crowds. Annual Review of Fluid Mechanics 35, 169-182.

Kapadia, M., Singh, S., Hewlett, W., and Faloutsos, P. 2009. Egocentric affordance fields in pedestrian steering. In Proc. 2009 Symposium on Interactive 3D graphics and games (I3D '09), ACM, New York, NY, USA, 215-223.

Karamouzas, I., Heil, P., van Beek, P., And Overmars, M. H. 2009. A predictive collision avoidance model for pedestrian simulation. In Motion in Games, 41-52.

Kuffner, J. J., J., AND Latombe, J. C. 1999. Fast synthetic vision, memory, and learning models for virtual humans. In Proc. Computer Animation, 118-127.

LAmarche, F., AND Donikian, S. 2004. Crowds of virtual humans : a new approach for real time navigation in complex and structured environments. Eurographics'04: Computer Graphics Forum 23, 3 (September), 509-518.

Loscos, C., Marchal, D., And Meyer, A. 2003. Intuitive crowd behaviour in dense urban environments using local laws. Theory and Practice of Computer Graphics (TPCG'03).

MASSIVE. http://www.massivesoftware.com. 
Narain, R., Golas, A., Curtis, S., And Lin, M. 2009. Aggregate dynamics for dense crowd simulation. In SIGGRAPH Asia '09: ACM SIGGRAPH Asia 2009 papers.

Noser, H., Renault, O., Thalmann, D., and Thalmann, N. M. 1995. Navigation for digital actors based on synthetic vision, memory, and learning. Computers \& Graphics 19, 1, 719. Computer Graphics Lab., Swiss Federal Inst. of Technol., Lausanne, Switzerland.

Paris, S., Donikian, S., And Bonvalet, N. 2006. Environmental abstraction and path planning techniques for realistic crowd simulation. CASA 2006: Computer Animation and Virtual Worlds 17, 3-4, 335.

Paris, S., Pettré, J., And Donikian, S. 2007. Pedestrian reactive navigation for crowd simulation: a predictive approach. Eurographics'07: Computer Graphics Forum 26, (3), 665-674.

Pelechano, N., Allbeck, J. M., And Badler, N. I. 2007. Controlling individual agents in high-density crowd simulation. In SCA '07: Proceedings of the 2007 ACM SIGGRAPH/Eurographics symposium on Computer animation, 99 108.

Pelechano, N., Allbeck, J., And Badler., N. I. 2008. Virtual Crowds: Methods, Simulation, and Control. Morgan \& Claypool Publishers. (Series Editor: Brian Barsky).

Peters, C., AND O'Sullivan, C. 2003. Bottom-up visual attention for virtual human animation. In International Conference on Computer Animation and Social Agents (CASA'03), 111-117.

Pettré, J., Ciechomski, P. D. H., Maïm, J., Yersin, B., LAUMOND, J.-P., AND THALMANN, D. 2006. Real-time navigating crowds: scalable simulation and rendering. Computer Animation and Virtual Worlds 17, 3-4, 445-455.

Pettré, J., Ondřej, J., Olivier, A.-H., Cretual, A., And DONIKIAN, S. 2009. Experiment-based modeling, simulation and validation of interactions between virtual walkers. In Proc. 2009 ACM SIGGRAPH/Eurographics Symposium on Computer Animation (SCA '09), ACM, New York, NY, USA, 189-198.

REYNOLDS, C. W. 1987. Flocks, herds and schools: A distributed behavioral model. In SIGGRAPH '87: Proceedings of the 14th annual conference on Computer graphics and interactive techniques, ACM, New York, NY, USA, 25-34.

REYNOLDS, C. W. 1999. Steering behaviors for autonomous characters. In Game Developers Conference 1999.

SCHADSCHNEIDER, A. 2001. Cellular automaton approach to pedestrian dynamicstheory. In In Pedestrian and Evacuation Dynamics, 75-85.

Shao, W., And Terzopoulos, D. 2005. Autonomous pedestrians. In Proc. 2005 ACM SIGGRAPH/Eurographics symposium on Computer animation (SCA '05), ACM Press, New York, NY, USA, 19-28.

Silva, A. R. D., Lages, W. S., and Chaimowicz, L. 2009. Boids that see: Using self-occlusion for simulating large groups on gpus. Comput. Entertain. 7, 4, 1-20.

Sud, A., Andersen, E., Curtis, S., Lin, M., And Manocha, D. 2007. Real-time path planning for virtual agents in dynamic environments. Proc. IEEE VR 2007, 91-98.

Thalmann, D., And Raupp Musse, S. 2007. Crowd Simulation. Springer, London.
Thalmann, D., Kermel, L., Opdyke, W., and Regelous, S. 2005. Crowd and group animation. In SIGGRAPH '05: ACM SIGGRAPH 2005 Courses, ACM, New York, NY, USA, 1.

Tresilian, J. R. 1994. Perceptual and motor processes in interceptive timing. Human Movement Science 13, 335-373.

Treuille, A., Cooper, S., And Popović, Z. 2006. Continuum crowds. ACM Transactions on Graphics (SIGGRAPH 2006) 25 , (3).

Tu, X., And Terzopoulos, D. 1994. Artificial fishes: physics, locomotion, perception, behavior. In SIGGRAPH '94: Proceedings of the 21st annual conference on Computer graphics and interactive techniques, ACM, New York, NY, USA, 43-50.

VAn Den Berg, J., Patil, S., Sewall, J., Manocha, D., AND LIN, M. 2008. Interactive navigation of individual agents in crowded environments. Symposium on Interactive $3 D$ Graphics and Games (I3D 2008).

Warren, W. H., and Fajen, B. R. 2004. Optic Flow and Beyond. Kluwer (Editors: L. M. Vaina, S. A. Beardsley, and S. Rushton), ch. From optic flow to laws of control, 307-337. 